\title{
GLOBAL STABILITY IN $n$-PERSON GAMES
}

\section{LOUIS J. BILLERA(1)}

\begin{abstract}
A class of bargaining sets, including the bargaining set $m_{1}^{(i)}$ and the kernel, is treated with regard to studying the tendency to reach stability from unstable points. A known discrete procedure is extended, and these results are applied to derive global stability properties for the solutions of certain differential equations. These differential equations are given in terms of the demand functions which define the bargaining sets, and the set of critical points is precisely the bargaining set in question.
\end{abstract}

1. Introduction. Most solution concepts that have been proposed for $n$-person games are essentially static in nature. That is, once a "stable" point is proposed, there will be no "objection" to that point, and the tendency will be to stay there. However, nothing is said about what is likely to occur at a point which is not stable. There is nothing generally in the definition of stability to guarantee that an unstable situation will tend toward a stable one.

In [14], Stearns showed that for a class of solution concepts related to the bargaining set $\mathbb{M}_{1}^{(i)}[10]$ and the kernel [5], one could define a discrete procedure which, starting with any payoff, converges to a stable point. In this paper, we extend the notion of transfer sequence and, relying heavily on Stearns' methods, prove the necessary convergence results.

We then consider the subclass of Stearns' class of bargaining sets which can be described by continuous demand functions. With each of these bargaining sets we associate a system of differential equations, the critical points of which are precisely the points in the bargaining sets. We prove that for any initial point, there is a solution to this system which approaches a stable point as $t \rightarrow \infty$. In case of the bargaining set $\pi_{1}^{(i)}$ and the kernel, these differential equations have unique solutions for any initial point.

Intuitively, these results have the following interpretation. At each payoff $x$, there is a number $d_{i j}(x)$ which measures the demand of player $i$ against player $j$. A point is stable if, for all pairs of players, the demands are zero. The differential

Received by the editors April 6, 1971. $34 \mathrm{D} 20$.

AMS (MOS) subject classifications (1970). Primary 90D12; Secondary 90D10, 34D05,

Key words and phrases. Cooperative games, kernel, bargaining sets, nucleolus, transfer schemes, global stability, asymptotic stability, characteristic function games, differential equations.

(1) This work was supported by the National Science Foundation under grant GK-4795, and partially by the Office of Naval Research under grant N00014-67-A-0077-0014. 
equations express the behavior that at any payoff $x$, all the players are acting to pay off all that they owe, and collect all they are owed. That is,

$$
x_{i}^{\prime}=\sum_{j} d_{i j}(x)-\sum_{j} d_{j i}(x) .
$$

It is this behavior that we prove leads to stability.

It is felt that these results are the first steps toward meeting the criticism that $n$-person game theory ignores the dynamic aspects of the situations it purports to model. It is hoped more results of this type will be forthcoming, in particular in such areas as the core, games without side payments, Nash equilibrium points and games with infinitely many players.

2. General transfer sequences. Let $n$ be a positive integer, and let $N=$ $\{1, \cdots, n\}$. Let $Y$ be a compact, convex polyhedron in $R^{n}$, real Euclidean $n$-space. We will denote by $R^{n \times n}$ the Euclidean space of points $p$ having $n^{2}$ coordinates, denoted $p_{i j}$ for all $i, j \in N$. We will also use the notation $[x]^{+}=\max (x, 0)$.

We define the function $\alpha: Y \rightarrow R^{n \times n}$ by the coordinate functions

$$
a_{i j}(x)=\left\{\begin{array}{l}
\sup \{\epsilon \mid x(\epsilon ; i, j) \in Y\} \quad \text { if } i \neq j, \\
0 \text { if } i=j,
\end{array}\right.
$$

where $x \in Y, x(\epsilon ; i, j)=\left(x_{1}, \cdots, x_{i}+\epsilon, \cdots, x_{j}-\epsilon, \cdots, x_{n}\right)$. We note here that $\alpha(x)$ is defined and nonnegative (where this means each coordinate is nonnegative) for each $x \in Y$. Since $Y$ is convex, it follows that $\alpha$ is a concave function on $Y$. It follows from linear programming theory that $\alpha$ is continuous and piecewise linear on $Y$.

Let $v$ be an $n$-person game, that is, let $v: 2^{N} \rightarrow R$ be such that $v(\varnothing)=0$. Thinking of points in $R^{2^{n}}$ as being indexed by subsets of $N$, we define $e: R^{n} \rightarrow$ $R^{2^{n}}$ by the coordinate functions $e_{S}(x)=v(S)-x(S)$, where $x(S)$ is used to denote $\Sigma_{i \in S} x_{i}, x(\varnothing)=0$. Now we define $s: R^{n} \rightarrow R^{n \times n}$ by

$$
s_{i j}(x)=\left\{\begin{array}{l}
\max \left\{e_{T}(x) \mid i \in T, j \notin T\right\} \text { if } i \neq j, \\
0 \quad \text { if } i=j .
\end{array}\right.
$$

Finally, we define $k: R^{n} \rightarrow R^{n \times n}$ and $k^{*}: Y \rightarrow R^{n \times n}$ by

$$
k_{i j}(x)=1 / 2\left[s_{i j}(x)-s_{j i}(x)\right]^{+} \text {and } k_{i j}^{*}(x)=\min \left(k_{i j}(x), \alpha_{i j}(x)\right) .
$$

Note that $k(x) \geq 0$ and $k^{*}(y) \geq 0$ for $x \in R^{n}$ and $y \in Y$.

It is easy to see that $\mathcal{K}(Y)=\left\{x \in Y \mid k^{*}(x)=0\right\}$ where $\mathcal{K}(Y)$ is the kernel of $Y$ (see [13] for the definitions). In particular, let $\mathscr{B}$ be a partition of $N$ and define

$$
Y_{\mathbb{B}}=\left\{x \in R^{n} \mid x_{i} \geq v(\{i\}), i \in N ; x(S)=v(S), S \in \mathbb{R}\right\} .
$$

Then $\mathcal{K}\left(Y_{B}\right)$ is the kernel for the coalition structure $\mathfrak{B}$ studied in [5], [6] and [7]. We remark here that $Y_{\mathscr{B}}$ is a compact, convex polyhedron (which, however, may be empty since we are assuming nothing about the game $v$ ). 
Let $\eta: R^{2^{n}} \rightarrow R^{2^{n}}$ be the ordering function, that is, the coordinates of $\eta(z)$ are just the coordinates of $z$ arranged in decreasing order: $\eta^{1}(z) \geq \eta^{2}(z) \geq \cdots \geq$ $\eta^{2^{n}}(z)$. We let $\theta: R^{n} \rightarrow R^{2^{n}}$ be the composition $\eta^{\circ} e$, and finally, for each $l=$ $1,2, \cdots, 2^{n}$, we let $\phi_{l}: R^{n} \rightarrow R$ be such that $\phi_{l}(x)=\sum_{i=1}^{l} \theta_{i}(x)$, for $x \in R^{n}$. For convenience, we define $\phi_{0} \equiv 0$.

Lemma 2.1. Let $x \in R^{n}$ and let $y=x(\epsilon ; i, j)$ where $0 \leq \epsilon \leq k_{i j}(x)$. Then for each $l=1, \cdots, 2^{n}$, there exists an integer $\bar{l}<l$ such that $\phi_{l}(y)-\phi_{l}(x) \leq$ $l\left(\phi_{\bar{l}}(x)-\phi_{\bar{l}}(y)\right)$.

Proof. If $\epsilon=0$, we may take $\bar{l}=0$, so we assume that $\epsilon>0$. Let $A=\{S \subset$ $\left.N \mid e_{S}(x) \geq s_{i j}(y)\right\}$, and let $l^{*}=|A|$. Suppose $s_{i j}(x)=e_{T}(x)$ and $s_{j i}(x)=e_{R}(x)$. From the definition of $k_{i j}$, it follows that

$$
s_{i j}(x)-\epsilon=s_{i j}(y) \geq s_{j i}(y)=s_{j i}(x)+\epsilon
$$

and therefore that $T \in A, R \notin A$. Thus for $l<l^{*}, \phi_{l}(y)-\phi_{l}(x) \leq 0$, while $\phi_{l^{*}}(y)-$ $\phi_{l^{*}}(x) \leq-\epsilon$. If $l \leq l^{*}$, the lemma follows by choosing $\bar{l}=0$. Since no coordinate of $e$ changes by more than $\epsilon$, we may choose $\bar{l}=l^{*}$ if $l>l^{*}$.

Lemma 2.2 Let $x, y^{1}, \ldots, y^{m} \in R^{n}$, and suppose that for each $i=1, \ldots, m$ and each $l=1, \cdots, 2^{n}$, there exists an integer $T<l$ such that

$$
\phi_{l}\left(y^{i}\right)-\phi_{l}(x) \leq l\left(\phi_{\bar{l}}(x)-\phi_{\bar{l}}\left(y^{i}\right)\right)
$$

Then if $y=(1 / m) \sum_{i=1}^{m} y^{i}$, we have, for each $l=1, \cdots, 2^{n}$,

$$
\phi_{l}(y)-\phi_{l}(x) \leq(m l)^{l} \sum_{0 \leq i<l}\left[\phi_{i}(x)-\phi_{i}(y)\right]^{+} .
$$

Proof. We first note that, for each $l, \phi_{l}(y) \leq(1 / m) \Sigma_{i=1}^{m} \phi_{l}\left(y^{i}\right)$. We may assume without loss of generality that we have equality for each $l$.

Suppose $\phi_{l}(y)-\phi_{l}(x)=\omega_{0}>0$. Then there is an index $i_{0}$ and an $l_{1}<l$ such that $\omega_{0} \leq \phi_{l}\left(y^{i 0}\right)-\phi_{l}(x) \leq l\left(\phi_{l_{1}}(x)-\phi_{l_{1}}\left(y^{i}\right)\right)$. Thus $\phi_{l_{1}}(x)-\phi_{l_{1}}\left(y^{i 0}\right) \geq \omega_{0} / l$. Let $\omega_{1}$ be such that $\left[\phi_{l_{1}}(x)-\phi_{l_{1}}(y)\right]^{+}=\omega_{0} / m l-\omega_{1} / m l$. If $\omega_{1}>0$, then there is an $i_{1}$ such that $\phi_{l_{1}}\left(y^{i} 1\right)-\phi_{l_{1}}(x) \geq \omega_{1} / m l$. Proceeding inductively, suppose $l_{j-1}, i_{j-1}$ and $\omega_{j-1}$ have been defined so that $\phi_{l_{j-1}}\left(y^{i j-1}\right)-\phi_{l_{j-1}}(x) \geq \omega_{j-1} /(m l)^{j-1}$. Then there is an $l_{j}<l_{j-1}$ such that

$$
\phi_{l_{j}}(x)-\phi_{l_{j}}\left(x^{i}{ }^{j-1}\right) \geq \omega_{j-1} /(m l)^{j-1} l_{j-1} \geq \omega_{j-1} / m^{j-1} l^{j} .
$$

Let $\omega_{j}$ be such that $\left[\phi_{l_{j}}(x)-\phi_{l_{j}}(y)\right]^{+}=\left(\omega_{j-1}-\omega_{j}\right) /(m l)^{j}$. If $\omega_{j}>0$, there is an $i_{j}$ such that $\phi_{l_{j}}\left(y^{i}\right)-\phi_{l_{j}}(x) \geq \omega_{j} /(m l)^{j}$.

Since $l>l_{1}>\cdots>l_{j} \geq 0$, we must eventually reach an integer $p \leq l$ such that $\omega_{p} \leq 0$. Then we have 


$$
\begin{aligned}
(m l)^{l} \sum_{0 \leq i<l}\left[\phi_{i}(x)\right. & \left.-\phi_{i}(y)\right]^{+} \geq(m l)^{l} \sum_{i=1}^{p}\left(\frac{\omega_{i-1}-\omega_{i}}{(m l)^{i}}\right) \\
& \geq \sum_{i=1}^{p}\left(\omega_{i-1}-\omega_{i}\right)=\omega_{0}-\omega_{p} \geq \omega_{0}=\phi_{l}(y)-\phi_{l}(x) .
\end{aligned}
$$

Lemma 23. There exists a real number $c$ such that for $x \in R^{n}$, if for each $i$, $j \in N, y^{i j}=x\left(\epsilon_{i j} ; i, j\right)$ where $0 \leq \epsilon_{i j} \leq k_{i j}(x)$, then if we set $y=\left(1 / n^{2}\right) \Sigma_{i, j \in N} y^{i j}$, we have

$$
\|y-x\| \equiv \sum_{i \in N}\left|y_{i}-x_{i}\right| \leq c \sum_{l=0}^{2^{n}}\left[\phi_{l}(x)-\phi_{l}(y)\right]^{+} .
$$

Proof. Since for $i \in N, y_{i}=x_{i}+\left(1 / n^{2}\right) \Sigma_{j \in N}\left(\epsilon_{i j}-\epsilon_{j i}\right)$, we have

$$
\begin{aligned}
\|y-x\| & =\frac{1}{n^{2}} \sum_{i \in N}\left|\sum_{j \in N}\left(\epsilon_{i j}-\epsilon_{j i}\right)\right| \\
& \leq \frac{2}{n^{2}} \sum_{i, j \in N} \epsilon_{i j} \leq 2 \max _{i, j \in N} \epsilon_{i j} .
\end{aligned}
$$

Suppose $\epsilon_{a b}=\max _{i, j \in N} \epsilon_{i j}>0$. As in the proof of Lemma 2.1, there is an $l$ such that $\phi_{l}\left(y^{a b}\right)-\phi_{l}(x) \leq-\epsilon_{a b}$. Let $\omega_{1}$ be such that $\left[\phi_{l}(x)-\phi_{l}(y)\right]^{+}=\left(\epsilon_{a b}-\omega_{1}\right) / n^{2}$. If $\omega_{1}>0$, then continue as in the proof of Lemma 2.2 to obtain

$$
\epsilon_{a b} \leq\left(n^{2} 2^{n}\right)^{2^{n}} \sum_{p=0}^{2^{n}}\left[\phi_{p}(x)-\phi_{p}(y)\right]^{+} .
$$

Setting $c=2\left(n^{2} 2^{n}\right)^{2^{n}}$, we have the desired result.

Lemma 2.4. Suppose for each $i, j \in N$, we are given $\epsilon_{i j}$ where $0 \leq \epsilon_{i j} \leq k_{i j}(x)$ for a fixed $x \in R^{n}$. Then $\Sigma_{j \in N}\left(\epsilon_{i j}-\epsilon_{j i}\right)=0$ for each $i \in N$ if and only if $\epsilon_{i j}=0$ for eacb $i, j \in N$.

Proof. Sufficiency is obvious. To prove necessity, notice first that if $i, j, l \epsilon$ $N$ then $k_{i j}(x)>0$ implies $k_{j i}(x)=0$, and $k_{i j}(x)>0$ and $k_{j l}(x)>0$ imply $k_{i l}(x)>0$. The proof of this follows almost exactly as in [5, Lemma 5.1].

Suppose for each $i \in N, \Sigma_{j \in N}\left(\epsilon_{i j}-\epsilon_{j i}\right)=0$, but $\epsilon_{i_{1} i_{2}}>0$. Thus $k_{i_{1} i_{2}}(x)>0$. By checking the above sum for $i=i_{2}$, we find an $i_{3}$ so that $\epsilon_{i_{2} i_{3}}>0$; hence $k_{i_{2} i_{3}}(x)>0$ which implies $k_{i_{1} i_{3}}(x)>0$. We may continue this process and obtain $i_{4}, \cdots, i_{p}$ so that $\epsilon_{i_{l} i_{l+1}}>0$ and $k_{i_{1} i_{l+1}}(x)>0, l=1, \cdots, p-1$. Since the number of indices is finite, we must eventually have some index appear twice. Assume without loss of generality that $i_{1}=i_{p}$. Then $k_{i_{1} i_{1}}(x)>0$, which is impossible. Therefore, $\epsilon_{i j}=0$ for all $i, j \in N$. 
Definition 2.5. Let $x \in R^{n}$. The $x$-core, $C(x)$, is given by $C(x)=\left\{y \in R^{n} \mid y(N)=\right.$ $\left.x(N) ; \theta_{1}(y) \leq \theta_{1}(x)\right\}$.

Note that for any $x, C(x)$ is a compact, convex polyhedron and $x \in C(x)$. We remark that if $x$ is a payoff vector, then $C(x)$ coincides with the strong $\theta_{1}(x)$-core for $\theta_{1}(x) \geq 0$, and $C(x)$ is the core when $x$ belongs to the core (i.e., when $\theta_{1}(x)=$ $0)$. (See [8].) It is easy to see that if $y \in C(x)$ then $C(y) \subset C(x)$.

Definition 2.6. Suppose $x \in R^{n}$, and for each $i, j \in N, 0 \leq \epsilon_{i j} \leq\left(1 / n^{2}\right) k_{i j}(x)$. Let $y \in R^{n}$ be defined by

$$
y_{i}=x_{i}+\sum_{j \in N}\left(\epsilon_{i j}-\epsilon_{j i}\right) \text { for } i \epsilon N
$$

Then we say $y$ results from $x$ by a k-bounded transfer.

We observe here that if $y$ results from $x$ by a $k$-bounded transfer, then if we set $\delta_{i j}=n^{2} \epsilon_{i j}$, we have $y=\left(1 / n^{2}\right) \Sigma_{i, j \in N} x\left(\delta_{i j} ; i, j\right)$. Thus, $y$ satisfies the hypotheses of Lemmas 2.2 and 2.3.

Lemma 2.7. If $y$ results from $x$ by a k-bounded transfer, then $y \in C(x)$.

Proof. It follows from the definition that $y(N)=x(N)$. Since $\phi_{1}=\theta_{1}$, the result follows from Lemma 2.2.

Defining $k^{*}$-bounded transfers in the obvious way, we can observe that if $x \epsilon$ $Y$ and $y$ results from $x$ by a $k^{*}$-bounded transfer, then $y \in C(x) \cap Y$.

We say a sequence $\left\{x^{i}\right\}_{i=0}^{\infty}$ of points in $R^{n}$ is a transfer sequence if, for each $i, x^{i+1}$ results from $x^{i}$ by a $k$-bounded transfer. By applying Lemma 2.7 , we can obtain the following result.

Lemma 2.8. If $\left\{x^{i}\right\}_{i=0}^{\infty}$ is a transfer sequence, then for each $i, x^{i} \in C\left(x^{0}\right)$. Thus every transfer sequence is bounded. Further, if $x^{0} \in Y$ and all the transfers are $k^{*}$. bounded, then $x^{i} \in C\left(x^{0}\right) \cap Y$ for each $i$.

We are able to say more. As we will next prove, every transfer sequence is convergent.

Theorem 2.9. Given $x^{0} \in R^{n}$, there exists a real number $\beta\left(x^{0}\right)$ such that, for all transfer sequences $\left\{x^{i}\right\}_{i=0}^{\infty}$ starting at $x^{0}$,

$$
\sum_{i=0}^{\infty}\left\|x^{i+1}-x^{i}\right\| \leq \beta\left(x^{0}\right) .
$$

Further, given a compact, convex set $Y$, there exists a real number $\beta(Y)$, independent of the game $v$ such that, for any $x^{0} \in Y$ and any sequence $\left\{x^{i}\right\}_{i=0}^{\infty}$ consisting only of $k^{*}$-bounded transfers, (2.9.1) is true with $\beta(Y)$ in place of $\beta\left(x^{0}\right)$.

Proof. For each $i$ and integer $l, 0 \leq l \leq 2^{n}$, set 


$$
\delta_{l}^{+}(i)=\left[\phi_{l}\left(x^{i+1}\right)-\phi_{l}\left(x^{i}\right)\right]^{+} \text {and } \delta_{l}^{-}(i)=\left[\phi_{l}\left(x^{i}\right)-\phi_{l}\left(x^{i+1}\right)\right]^{+} \text {. }
$$

In addition let $I_{l}^{r}=\Sigma_{i=0}^{r} \delta_{l}^{+}(i)$ and $D_{l}^{r}=\Sigma_{i=0}^{r} \delta_{l}^{-}(i)$. Then by Lemma 2.3, $\| x^{i+1}-$ $x^{i} \| \leq c \Sigma_{l=0}^{2^{n}} \delta_{l}^{-}(i)$, and thus

$$
\sum_{i=0}^{r}\left\|x^{i+1}-x^{i}\right\| \leq c \sum_{l=0}^{2^{n}} D_{l}^{r}
$$

We will show the existence of a $\beta\left(x^{0}\right)$ (or $\beta(Y)$, in the second case) such that, for all $r \geq 0, c \Sigma_{l=0}^{2^{n}} D_{l}^{r} \leq \beta\left(x^{0}\right)$ (or $\beta(Y)$ ).

By Lemma 2.2, we have for each $l, \delta_{l}^{+}(i) \leq\left(n^{2} l\right)^{l} \Sigma_{0<_{j}<l} \delta_{j}^{-}(i)$. Therefore, for all $r \geq 0$, by summing from 0 to $r$, we get

$$
I_{l}^{r} \leq\left(n^{2} l\right)^{l} \sum_{0 \leq j<l} D_{l}^{r}
$$

Now $\delta_{l}^{-}(i)-\delta_{l}^{+}(i)=\phi_{l}\left(x^{i}\right)-\phi_{l}\left(x^{i+1}\right)$, so

$$
D_{l}^{r}-I_{l}^{r}=\phi_{l}\left(x^{0}\right)-\phi_{l}\left(x^{r+1}\right) \text {. }
$$

By Lemma 2.8, both $x^{0}, x^{r+1} \in C\left(x^{0}\right)$ (or $C\left(x^{0}\right) \cap Y$, for the second case). By the compactness of $C\left(x^{0}\right)$ (or $Y$ ), there exists a $\rho\left(x^{0}\right)(\rho(Y)$ ) such that

$$
\max _{S \subseteq N}|x(S)-y(S)| \leq \rho\left(x^{0}\right) \quad(\rho(Y))
$$

for all $x, y \in C\left(x^{0}\right)(x, y \in Y)$. Thus for $S \subseteq N, e_{S}(y)-e_{S}(x) \leq \rho\left(x^{0}\right)$, and thus $\phi_{l}(y)-\phi_{l}(x) \leq l \rho\left(x^{0}\right)$ for $x, y \in C\left(x^{0}\right)$ (or $\phi_{l}(y)-\phi_{l}(x) \leq l \rho(Y)$ for $\left.x, y \in Y\right)$. Applying this to (2.9.3), we get for each $l$

$$
D_{l}^{r} \leq I_{l}^{r}+l \rho\left(x^{0}\right) \quad(l \rho(Y))
$$

Note here that $\rho\left(x^{0}\right)$ depends on $C\left(x^{0}\right)$, therefore on $x^{0}$ and $v$. On the other hand, $\rho(Y)$ depends only on $Y$.

To derive the bounds $\beta\left(x^{0}\right)$ and $\beta(Y)$, notice first that, for all $r \geq 0, I_{0}^{r}=D_{0}^{r}=$ 0 . Now suppose that $I_{j}^{r}$ and $D_{j}^{r}$ have been given bounds independent of $r$ for $j=$ $0, \cdots, l-1$. First (2.9.2) provides a bound for $I_{l}^{r}$, and then (2.9.4) provides a bound for $D_{l}^{r}$. Both these bounds are independent of $r$. The theorem follows.

3. Demand functions and bargaining sets. By a demand function, we will mean a continuous function $d: R^{n} \rightarrow R^{n \times n}$ which satisfies $0 \leq d(x) \leq k(x)$ for all $x \in R^{n}$.

For a given demand function $d$, we define a function $D: R^{n} \rightarrow R^{n}$ by $D_{i}=$ $\Sigma_{j \in N}\left(d_{i j}-d_{j i}\right)$. It follows from Lemma 2.4 that $D(x)=0$ if and only if $d_{i j}(x)=0$ for all $i, j \in N$. We now can define the bargaining set associated with the demand function $d$ as

$$
M(d)=\left\{x \in R^{n} \mid D(x)=0\right\} .
$$


This agrees with the definition given by Stearns in [14].

Suppose, in addition, that $d(y) \leq k^{*}(y)$ for $y \in Y$. Then we say $d$ is $k^{*}$ bounded and we define $M_{Y}(d)=M(d) \cap Y$. The Brouwer fixed point theorem shows that $M_{Y}(d)$ $\neq \varnothing$ since the map $f: Y \rightarrow Y$ given by $f(x)=x+\left(1 / n^{2}\right) D(x)$ must have a fixed point. The nonemptiness of $M(d)$ for general $d$ will follow from the next result.

Theorem 3.1. Let $x^{0} \in R^{n}$ and suppose a sequence $\left\{x^{i}\right\}_{i=0}^{\infty}$ is defined by $x^{i+1}$ $=x^{i}+b D\left(x^{i}\right)$ where $0<b \leq 1 / n^{2}$. Then $\left\{x^{i}\right\}$ converges to a point $x \in M(d)$.

Further, if $x^{0} \in Y$ and $d$ is $k^{*}$ bounded, then $\left\{x^{i}\right\}$ converges to $x \in M_{Y}(d)$.

Proof. In either case, the sequence is a transfer sequence, and therefore Theorem 2.9 applies to give $\sum_{i=0}^{\infty}\left\|x^{i+1}-x^{i}\right\|<\infty$. This implies the sequence is Cauchy, since for $m>k$,

$$
\left\|x^{m}-x^{k}\right\| \leq \sum_{i=k}^{m-1}\left\|x^{i+1}-x^{i}\right\| \leq \sum_{i=k}^{\infty}\left\|x^{i+1}-x^{i}\right\|,
$$

which can be made small for large $k$. Thus there exists $x \in R^{n}$ s.t. $x^{i} \rightarrow x$. Since $D$ is continuous, and $\left\|D\left(x^{i}\right)\right\|=b^{-1}\left\|x^{i+1}-x^{i}\right\| \rightarrow 0$, it follows that $D(x)=0$ and $x \in M(d)$ in the first case.

In the second case, Lemma 2.8 implies that $x \in Y$ and by the above argument, $D(x)=0$.

Corollary 3.2 $M(d) \neq \varnothing$, in fact, for any $x^{0} \in R^{n}$, there exists an $x \in M(d) \cap$ $C\left(x^{0}\right)$. If $d$ is $k^{*}$ bounded, then for any $x^{0} \in Y$, there exists an $x \in M_{Y}(d) \cap C\left(x^{0}\right)$.

We remark here that in either of the cases treated in 3.1 we have $\sum_{i=0}^{\infty}\left\|x^{i+1}-x^{i}\right\|<\beta$, where $\beta$ will depend either on $x^{0}$ or on $Y$, but $\beta$ is independent of $b$.

4. Global stability. Let $d$ be a demand function, and consider the system of differential equations

$$
x^{\prime}=D(x) \text {. }
$$

Since $D$ is continuous, there exist solutions to (4.1) through any initial point $x^{0} \epsilon$ $R^{n}$. From [9, Theorem 1.31, p. 9], it follows that the solutions of (4.1) are defined for all $t \in R$, since each $D_{i}$ is nonnegative and bounded above by the piecewise linear function $\Sigma_{j \in N}\left(k_{i j}+k_{j i}\right)$.

We will be concerned here only with solutions defined on $R_{+}=[0, \infty)$ and starting at some fixed inital point, $x^{0} \in R^{n}$. That is, we will consider functions $\phi: R_{+} \rightarrow R^{n}$ such that $\phi(0)=x^{0}$ and $d \phi_{i}(t) / d t=D_{i}(\phi(t))$ for all $t \in R_{+}$.

Theorem 4.1. For any $x^{0} \in R^{n}$, there exists a solution $\phi$ to $x^{\prime}=D(x)$ such that $\phi(0)=x^{0}$ and $\phi(t)$ converges to a point $x \in M(d)$ as $t \rightarrow \infty$. If, in addition, $x^{0} \in Y$ and $d$ is $k^{*}$ bounded, then $\phi(t) \in Y$ for all $t \in R_{+}$and $x \in M_{Y}(d)$. 

$C\left(x^{0}\right)$.

Proof. Let $x^{0} \in R^{n}$, and choose a real number $M$ so that $\|D(x)\| \leq M$ for $x \in$

Let $0<b \leq b_{0}=1 / n^{2}$, and define the sequence $\left\{x^{i}\right\}$ by $x^{i+1}=x^{i}+b D\left(x^{i}\right)$. Notice that $\left\{x^{i}\right\} \subset C\left(x^{0}\right)$, and if $x^{0} \in Y$ and $d$ is $k^{*}$ bounded, then $\left\{x^{i}\right\} \subset C\left(x^{0}\right) \cap$ $Y$, by Lemma 2.8. Now define $\phi^{b}: R_{+} \rightarrow C\left(x^{0}\right)$ by $\phi^{b}(0)=x^{0}$, and $\phi^{b}(t)=x^{i}+$ $D\left(x^{i}\right)(t-i b)$ where $i$ is chosen so that $i b<t \leq(i+1) b$. That $\phi^{b}$ maps into $C\left(x^{0}\right)$ follows from the above comments and the convexity of $C\left(x^{0}\right)$. Again, if $x^{0} \in Y$ and $d$ is $k^{*}$ bounded, then $\phi^{b}$ maps into $C\left(x^{0}\right) \cap Y$.

Let $\langle b\rangle=\{i b \mid i=0,1,2, \cdots\}$. On $R_{+}-\langle b\rangle$ we have that $\left(\phi^{b}\right)^{\prime}$ exists. (2) In fact, for $i b<t<(i+1) b,\left(\phi^{b}\right)^{\prime}(t)=D\left(x^{i}\right)$.

Let $\epsilon>0$, and choose $0<b_{\epsilon} \leq b_{0}$ so that, for $x, y \in C\left(x^{0}\right),\|y-x\|<M b_{\epsilon}$ implies $\|D(y)-D(x)\|<\epsilon$. Let $0<b<b_{\epsilon}, t \in R_{+}-\langle b\rangle$, and suppose $i b<t<(i+1) b$. Then since $\left\|\phi^{b}(t)-x^{i}\right\|=\left\|D\left(x^{i}\right)\right\||t-i b|<M b_{\epsilon}$, we have

$$
\left\|\left(\phi^{b}\right)^{\prime}(t)-D\left(\phi^{b}(t)\right)\right\|=\left\|D\left(x^{i}\right)-D\left(\phi^{b}(t)\right)\right\|<\epsilon .
$$

Clearly, $\left\|\phi^{b}(t)-\phi^{b}\left(t^{\prime}\right)\right\| \leq M\left|t-t^{\prime}\right|$ for all $0<b \leq b_{0}$. Thus the family of functions $\left\{\phi^{b} \mid 0<b \leq b_{0}\right\}$ is uniformly bounded and equicontinuous. Let $\left\{b_{j}\right\}_{j=1}^{\infty}$ be such that $b_{j} \leq b_{0}$ for each $j$ and $b_{j} \rightarrow 0$ as $j \rightarrow \infty$. Denote $\phi^{b_{j}}$ by $\phi^{j}$. By the Ascoli theorem, there is a subsequence of the $\phi^{j}$ (we may assume now without loss of generality that it is the entire sequence $\phi^{j}$ ) and a continuous function $\phi: R_{+} \rightarrow$ $C\left(x^{0}\right)$ such that $\phi^{j} \rightarrow \phi$ pointwise on $R_{+}$as $j \rightarrow \infty$ and the convergence is uniform on compact subsets of $R_{+}$. We show first that $\phi$ is a solution to $x^{\prime}=D(x)$. Clearly, $\phi(0)=x^{0}$. Note that $\left\|\phi(t)-\phi\left(t^{\prime}\right)\right\| \leq M\left|t-t^{\prime}\right|$.

Let $\Delta^{j}(t)=\left(\phi^{j}\right)^{\prime}(t)-D \circ \phi^{j}(t)$ for $t \in R$, such that $\left(\phi^{j}\right)^{\prime}(t)$ exists. Otherwise, let $\Delta^{j}(t)=0$. Thus, $D \circ \phi^{j}(t)+\Delta^{j}(t)=\left(\phi^{j}\right)^{\prime}(t)$ whenever $\left(\phi^{j}\right)^{\prime}(t)$ exists, that is, except for a countable subset of $R_{+}$. Since $\phi^{j}$ is absolutely continuous, it follows that

$$
\phi^{j}(t)=x^{0}+\int_{0}^{t}\left[D \circ \phi^{j}(\tau)+\Delta^{j}(\tau)\right] d \tau
$$

Since $\phi^{j} \rightarrow \phi$ uniformly on $[0, t]$ and $D$ is uniformly continuous on $C\left(x^{0}\right)$, we have that $D \circ \phi^{j} \rightarrow D \circ \phi$ uniformly on $[0, t]$. From the discussion above, it follows that $\Delta^{j}(t) \rightarrow 0$ uniformly on $R_{+}$. Thus

$$
\phi(t)=x^{0}+\int_{0}^{t} D \circ \phi(\tau) d \tau
$$

and finally $\phi^{\prime}(t)=D(\phi(t))$.

We show now that $\phi(t)$ converges to a point $x \in M(d)$ as $t \rightarrow \infty$. It follows from the remark following Corollary 3.2 that there is a number $\beta$ (independent of $b$ ) such

(2) $\left(\phi^{b}\right)^{\prime}=d \phi^{b} / d t$. 
that the total variation of the function $\phi^{b}, 0<b \leq b_{0}$, on the interval $[0, T]$ is less than $\beta$ for all $T \in R_{+}$. Since $\phi^{j} \rightarrow \phi$ it follows that the total variation of $\phi$ on $[0, T]$ is also less than $\beta$ for all $T$. Therefore, $\phi(t)$ converges to a unique limit point $x \in C\left(x^{0}\right)$ as $t \rightarrow \infty$.

To see that $x \in M(d)$, note that $D \circ \phi(t) \rightarrow D(x)$ as $t \rightarrow \infty$. For each integer $l \geq 0$, and for each $i=1, \cdots, n$, there is a $\tau_{i}^{l} \in R_{+}$, such that $l \leq \tau_{i}^{l} \leq l+1$ and

$$
\phi_{i}(l+1)-\phi_{i}(l)=\phi_{i}^{\prime}\left(\tau_{i}^{l}\right)=D_{i} \circ \phi_{i}\left(r_{i}^{l}\right) .
$$

Since for each $i, \tau_{i}^{l} \rightarrow \infty$ as $l \rightarrow \infty$, we have

$$
D_{i} \circ \phi\left(\tau_{i}^{l}\right) \rightarrow D_{i}(x) \text { as } l \rightarrow \infty
$$

But $\phi_{i}(l+1)-\phi_{i}(l) \rightarrow 0$ as $l \rightarrow \infty$ for each $i$, thus $D(x)=0$ and $x \in M(d)$.

If $x^{0} \in Y$ and $d$ is $k^{*}$ bounded, we have $\phi\left(R_{+}\right) \subset C\left(x^{0}\right) \cap Y$ since this holds for each $\phi^{b}$. It is clear that $x$ must be in $M_{Y}(d)$ in this case. This completes the proof.

We remark that in the case where $x^{0} \in Y$ and the demand functions are $k^{*}$ bounded, it is enough to consider them as if they were defined only on $Y$. This causes no loss of generality since any function $d^{*}: Y \rightarrow R^{n \times n}$ satisfying $d^{*} \leq k^{*}$ can be extended to a $k^{*}$-bounded demand function $d: R^{n} \rightarrow R^{n \times n}$ such that $\left.d\right|_{Y}=$ $d^{*}$.

5. Some special cases. If the demand function $d$ has the property that the system $x^{\prime}=D(x)$ has a unique solution through each initial point $x^{0}$, then Theorem 4.1 becomes particularly useful. A simple condition which guarantees uniqueness is that each of the functions $d_{i j}$ is piecewise linear with a finite number of different linear "pieces". Indeed, this condition implies that $D(x)$ is uniformly Lipschitz and hence that solutions of $x^{\prime}=D(x)$ are unique (see [4]).

It is clear that the function $k$ is piecewise linear on $R^{n}$, and since $Y$ is a polyhedron, $k^{*}$ is piecewise linear on $Y$. Letting $Y=Y_{\dot{B}}$ for some partition $\mathfrak{B}$ of $N$ (and assuming $Y \neq \varnothing$ ), we have $K(Y)=M_{Y}\left(k^{*}\right)$ which is the kernel for the coalition structure $\mathfrak{B}$.

Following Stearns [14], we can define a $k^{*}$-bounded demand function $m$ on $Y_{\mathscr{B}}$ so that $M_{Y}(m)$ is the bargaining set $\mathbb{M}_{1}^{(i)}$ for the coalition structure $\mathfrak{B}$ (see [10]). It can be shown that $m$ is also piecewise linear. The continuity of $m$ follows from [1, Lemma 2.11]. Taking these facts together with Theorem 4.1, we can state the following.

Theorem 5.1. The differential equations which describe (in the sense of \$4) the kernel $\mathcal{K}$ and the bargaining set $\mathbb{M}_{1}^{(i)}$ for any partition $\mathfrak{B}$ bave unique solutions through any point $x^{0} \in Y_{\mathscr{B}}$. Further, these solutions approach $\mathcal{K}$ and $\Re_{1}^{(i)}$ as $t \rightarrow \infty$. 
In the case where $v$ is monotonic in the zero normalization (see [8]) we can simplify the differential equations which describe the kernel $\mathcal{K}$ for the grand coalition (i.e. $B=\{N\}$ ).

Proposition 5.2 Let $Y=Y_{\{N\}}$. Then if $v$ is monotonic in the zero normalization, we bave

$$
\mathcal{K}=M_{Y}\left(k^{*}\right)=M(k) \cap\left\{x \in R^{n} \mid x(N)=v(N)\right\} .
$$

Proof. That $K=M_{Y}\left(k^{*}\right)$ is clear. We prove the second equality. The inclusion

$$
M_{Y}\left(k^{*}\right) \subset M(k) \cap\left\{x \in R^{n} \mid x(N)=\grave{v}(N)\right\}
$$

is Lemma 3.4 of [8]. To show the opposite inclusion, (3) suppose $x(N)=v(N)$ and $s_{i j}(x)=s_{j i}(x)$ for all $i, j \in N$, but $x_{k}<v(\{k\})$ for some $k$. Let $\mathfrak{D}$ be the set of coalitions $S$ for which $e_{S}(x)$ is maximum. Since $e_{\{k}(x)>0, N \notin \mathscr{D}$. Further, $S \in \mathcal{D}$ implies $k \in S$ since $T \in \mathscr{D}, k \notin T$ implies $e_{R}(x)>e_{T}(x)$, where $R=T \cup\{k\}$, by monontonicity in the zero normalization and the assumption that $x_{k}<v(\{k\})$. Therefore, there exists an $l \in N$ s.t. $s_{k l}(x)>s_{l k}(x)$, which is impossible. Thus, $x_{k} \geq v(\{k\})$.

Theorem 5.3. Let $v$ be monotonic in the zero normalization and let $\mathcal{K}$ be the kernal of $v$ for the grand coalition structure. Suppose $x^{0} \in R^{n}$ and $x^{0}(N)=v(N)$. Then the unique solution $\phi$ to $x^{\prime}=K(x)$ such that $\phi(0)=x^{0}$ bas the property that $\lim _{t \rightarrow \infty} \phi(t) \in \mathcal{K}$.

Proof. This is immediate from Theorem 4.1 and Proposition 5.2.

6. Comments. One problem left by this study is to obtain a direct proof of Theorem 4.1 , that is, one which does not require going through discrete transfer methods (at least in the case where the differential equations have unique solutions). It would seem from the methods of this study that one should be able to obtain stability theorems for systems of differential equations by use of lexicographically decreasing vector "Lyapunov functions". The function $\theta$ seems to serve this purpose here. However, the fact that the lexicographic ordering is not continuous keeps the standard proofs from working in this case.

It would be of great interest in Mathematical Economics if global stability results could be obtained for the core. Such results would say something about the tendency of an economy to reach equilibrium. A global stability result for the nucleolus (see [13]) would essentially be a result for the core since the nucleolus is in the core whenever the core is nonempty. We feel it is likely that the results here can be extended in some way to obtain convergence to the nucleolus.

It would also be interesting to extend the notion of a "behavioral vector field" to games without side payments and games with infinitely many players. In the case

(3) The author is indebted to Elon Kohlberg for this proof. 
of infinitely many players, it has so far been difficult even to extend the notion of bargaining set, much less to worry about existence. Perhaps the differential equations approach will prove more fruitful. Results on games without side payments ([11], [2] and [1]) seem to indicate that static stability may not always be possible, and that we may have to settle for some sort of cyclic behavior. Perhaps this can be formalized in terms of periodic solutions to related differential equations.

Finally, one can describe the Nash equilibrium point solutions to a multiperson noncooperative game by demand functions, and, therefore, by a system of differential equations. These equations resemble (but are essentially different than) the differential equations studied by Brown and von Neumann [3] for solving zero-sum two-person games. The notion of demand functions for equilibrium points is motivated by Peleg's treatment of equilibrium points as solutions to open acyclic relations in [12].

Roughly, let $S_{k}$ be the (finite) set of pure strategies for player $k, k=1, \cdots, m$. Let $n=\sum_{k=1}^{m}\left|S_{k}\right|$, and let

$$
Y=\left\{x \in R^{n} \mid x_{i} \geq 0 ; \sum_{i \in S_{k}} x_{i}=1, k=1, \cdots, n\right\}
$$

be the set of vectors of mixed strategies for the $m$ players. For each $k=1, \cdots, m$, let $H_{k}: Y \rightarrow R$ be the payoff function for player $k$ and let $H_{k}(x \mid i)$ denote the payoff to player $k$ when every other player plays his mixed strategy as given in $x$, but player $k$ uses his pure strategy $i$. Let $i, j \in N=\bigcup_{k=1}^{m} S_{k}$. We define the demand function $d: Y \rightarrow R^{n \times n}$ by $d_{i j}(x)=x_{j}\left[H_{k}(x \mid i)-H_{k}(x \mid j)\right]^{+}$when $i, j \in S_{k}$ for some $k$. Otherwise, $d_{i j}(x)=0$. It is clear that $x$ is an equilibrium point if and only if $d_{i j}(x)=0$ for all $i, j \in N$. It is not known whether Theorem 4.1 is true for the system $x^{\prime}=D(x)$ defined by the function $d$.

The author is grateful to M. Balch, M. Brown, C. Dafermos and M. Maschler for many helpful conversations on this work.

\section{REFERENCES}

1. N. Asscher, A bargaining set for cooperative games without side payments, Research Prog. in Game Theory and Math. Eco., Dept. of Math., The Hebrew University of Jerusalem, RM No. 58, April, 1970.

2. L. J. Billera, Existence of general bargaining sets for cooperative games without side payments, Bull. Amer. Math. Soc. 76 (1970), 375-379. MR 41 \#3117.

3. G. W. Brown and J. von Neumann, Solutions of games by differential equations, Contributions to the Theory of Games, Ann. of Math. Studies, no. 24, Princeton Univ. Press, Princeton, N. J., 1950, pp. 73-79. MR 12, 514.

4. E. A. Coddington and N. Levinson, Theory of ordinary differential equations, McGrawHill, New York, 1955. MR 16, 1022.

5. M. Davis and M. Maschler, The kernel of a cooperative game, Naval Res. Logist. Quart. 12 (1965), 223-259. MR 34 \#7219.

6. M. Maschler and B. Peleg, A characterization, existence proof and dimension bounds for the kernel of a game, Pacific J. Math. 18 (1966), 289-328. MR 34 \#5525. 
7. - The structure of the kernel of a cooperative game, SIAM J. Appl. Math. 15 (1967), 569-604. MR 3.7 \#183.

8. M. Maschler, B. Peleg and L. S. Shapley, The kernel and the nucleolus of a cooperative game as locuses in the stron $g$-core, Research Prog. in Game Theory and Math. Eco., Dept. of Math., The Hebrew University of Jerusalem, RM No. 60, May, 1970.

9. V. V. Nemyckiř and V. V. Stepanov, Qualitative theory of differential equations, OGIZ, Moscow, 1947; English transl., Princeton Math. Series, no. 22, Princeton Univ. Press, Princeton, N. J., 1960. MR 10, 612; MR 22 \#12258.

10. B. Peleg, Existence theorem for the bargaining set $M^{(i)}$, Essays in Mathematical Economics, Princeton Univ. Press, Princeton, N. J., 1967; cf. Bull. Amer. Math. Soc. 69 (1963), 109-110. MR 26 \#2333; MR 34 \#8826.

11. - Bargaining sets of cooperative games without side payments, Israel $\mathrm{J}$. Math. 1 (1963), 197-200. MR 30 \#2944.

12. - Equilibrium points for open acyclic relations, Canad. J. Math 19 (1967), 366-369. MR $34 \# 8825$.

13. D. Schmeidler, The nucleolus of a characteristic function game, SIAM J. Appl. Math. 17 (1969), 1163-1170. MR 41 \#5058.

14. R. E. Stearns, Convergent transfer schemes for $N$-person games, Trans. Amer. Math. Soc. 134 (1968), 449-459. MR 37 \#6112. 14850

DEPARTMENT OF OPERATIONS RESEARCH, CORNELL UNIVERSITY, ITHACA, NEW YORK 